\title{
DESEMPENHO DE LONGEVOS CAIDORES E NÃO CAIDORES NA AVALIAÇÃO DO TIMED UP AND GO UTILIZANDO UM APLICATIVO DE SMARTPHONE
}

\section{Gabriela Guimarães Oliveira}

Mestre em Gerontologia Biomédica pela Pontifícia Universidade Católica do Rio Grande do Sul - PUCRS, Porto Alegre, RS, Brasil.

\section{Márcio Sarroglia Pinho}

Doutor em Interação Colaborativa e Sistemas de Realidade Virtual pela Universidade Federal do Rio Grande do Sul. Coordenador de pesquisa da Escola Politécnica da PUCRS. Coordena o Centro de Pesquisa em Visualização Avançada da PUCRS, Brasil.

\section{Ângelo José Gonçalves Bós}

Doutor Phd In Medicine pela Tokai University, Japão. Pós-doutorado pelo Instituto Nacional Americano sobre o Envelhecimento e Escola de Saúde Pública da Universidade de Johns Hopkins em Baltimore, EUA. Pós-doutorado no Departamento de Saúde e Participação Social do Tokyo Metropolitan Institute of Gerontology, Japão.
Autor correspondente

Gabriela Guimarães

oliveira_gabriela@hotmail.com
RESUMO: Este estudo tem por objetivo observar o desempenho de longevos caidores e não caidores por meio do teste Timed Up and Go (TUG) utilizando um aplicativo de smartphone (Mobility Suite $\left.{ }^{\circledR}\right)$. Participaram longevos ( $\geq 90$ anos), de Porto Alegre (RS), acompanhados em domicílio. Cada longevo realizou o TUG utilizando um smartphone com sistema operacional Android que foi colocado em uma cinta elástica com velcro e preso no peito, na altura do esterno. Assim, parâmetros das fases individuais do teste foram fornecidos pelo aplicativo, sendo eles: a) duração total do TUG em segundos; b) duração da transição de sentado para em pé em segundos; c) duração da transição de pé para sentado em segundos; d) variação máxima do ângulo do tronco na fase de inclinação para frente em graus e; e) velocidade angular máxima durante a fase de inclinação para frente em graus/ segundos. Análise de variáveis sociodemográficas e clínicas, presença de multimorbidades e polifarmácia e variáveis relacionadas às quedas foram utilizadas para comparações. Dos 98 avaliados, 26,5\% referiram quedas nos últimos seis meses. Longevos caidores apresentaram mais sintomas depressivos $(\mathrm{p}<0,001)$. Caidores apresentaram pior desempenho em cada uma das fases do TUG de acordo com o aplicativo. O aplicativo de smartphone Mobility Suite ${ }^{\circledR}$ foi capaz de avaliar o desempenho de longevos caidores e não caidores por meio do tempo do TUG e seus parâmetros. A depressão mostrou-se a única variável estatisticamente significativa, indicando que longevos com sintomas depressivos apresentam mais chances de cair. Este foi o primeiro relato da utilização do MobilitySuite ${ }^{\circledR}$ em longevos. $\mathrm{O}$ aplicativo mostrou-se útil para utilização no ambiente domiciliar, podendo ser utilizado em investigações futuras.

PALAVRAS-CHAVE: Acidentes por quedas; Idoso de 80 anos ou mais; Saúde pública; Tecnologia.

\section{PERFORMANCE OF VERY ELDERLY FALLERS AND NON-FALLERS EVALUATED BY TIMED-UP-AND-GO WITH SMARTPHONE APP}

\begin{abstract}
The performance of very elderly people, fallers and non-fallers, is evaluated by the Timed Up and Go (TUG) test with smartphone app (Mobility Suite ${ }^{\circledR}$ ). Elderly people ( $\geq 90$ years) from Porto Alegre, Brazil, were monitored at home. Each one performed TUG using smartphone with Android operational system placed within an elastic belt and tied on the breast near the sternum. The parameters of the test's individual phases were provided by the app, namely, a) total duration of TUG, in seconds; b) duration of sitting-standing transition, in seconds; c) duration of standing-sitting, in seconds; d) maximum variation of the trunk angle within the inclination phase forward, in degrees; e) maximum angle speed during the inclination phase forward in degrees/seconds. Analysis of socio-demographic and clinical
\end{abstract}


variables, multi-morbidities and poly-pharmacy and variables related to falls were compared. Further, $26.5 \%$ of 98 very elderly people evaluated mentioned falls during the previous six months. Elderly people with falls had more depression symptoms $(p<0.001)$. According to app, fallers had the worst performance in each TUG phase. Smartphone Mobility Suite ${ }^{\circledR}$ app evaluated the performance of very elderly fallers and non-fallers during TUG and its parameters. Depression was the only variable statistically significant and indicated that very elderly people with depression had better chances for falling. This paper presents the first report in the use of MobilitySuite ${ }^{\circledR}$ for very elderly people. In fact, app was highly useful for use in the home and may be further used successfully.

KEY WORDS: Accidents by falling; Elderly people 80 years old or older; Public health; Technology.

\section{INTRODUÇÃO}

O envelhecimento populacional é considerado um fenômeno mundial ${ }^{1}$. O processo de envelhecimento vem acompanhado de alterações fisiológicas, que associadas às modificações gradativas e progressivas podem causar limitação funcional. Essas alterações são sobrepostas às doenças crônicas pré-existentes, que contribuem para o declínio da mobilidade funcional e consequentemente para a ocorrência de quedas ${ }^{1}$.

A queda é considerada um dos graves problemas de saúde pública. Aproximadamente 35 a $40 \%$ das pessoas acima de 65 anos sofrem pelo menos uma queda por ano e sua prevalência aumenta com a idade, chegando a $50 \%$ acima dos 80 anos $^{3}$. A queda é caracterizada como uma síndrome geriátrica complexa, de natureza multifatorial, que compromete a autonomia e a capacidade funcional do idoso, influenciando diretamente na sua qualidade de vida ${ }^{2}$.

Vários testes têm sido utilizados para avaliar o controle postural de idosos, a fim de direcionar tratamentos específicos para prevenção de quedas. Dentre eles, o TUG se destaca por ser de ampla aplicabilidade no contexto clínico e científico. Trata-se de um teste amplamente utilizado na população idosa, no entanto, pouco explorado na população longeva brasileira ${ }^{3}$.

As tecnologias de informação móveis encontram-se entre os principais temas discutidos atualmente no meio científico, e sua utilidade vem proporcionando o surgimento de uma série de questões relacionadas às suas vantagens no âmbito da saúde ${ }^{6}$. O desenvolvimento de tecnologias móveis tem propiciado a utilização das mesmas no contexto clínico. Dentre os dispositivos móveis utilizados atualmente, os smartphones se destacam, por serem equipamentos facilitadores em atividades que exigem mobilidade e praticidade ${ }^{4}$. Neste contexto, a proliferação de smartphones que integram um número crescente de sofisticados sensores cria uma série de oportunidades para quantificação e monitoramento das atividades humanas e procedimentos de diagnóstico5.

$\mathrm{Na}$ avaliação do risco de queda, Milosevic et al. ${ }^{6}$ intitulam-se um dos primeiros a propor e implementar um aplicativo de smartphone que automatiza e quantifica o teste TUG (Mobility Suite ${ }^{\circledR}$ ), introduzindo parâmetros que melhoram a sua caracterização ao levar em consideração as transições de postura corporal. Este aplicativo quantifica as fases do teste para permitir que profissionais da saúde possam avaliar melhor a cinemática e dinâmica do corpo, assim como, para que cuidadores e profissionais de saúde possam obter percepções sobre o bem-estar geral dos indivíduos ${ }^{6}$.

Dessa forma, sabendo-se da heterogeneidade da população idosa e das peculiaridades pertinentes a cada subgrupo etário, este estudo tem por objetivo avaliar o desempenho de longevos caidores e não caidores por meio da avaliação do teste TUG utilizando o aplicativo de smartphone Mobility Suite $\AA_{\text {. }}$

\section{METODOLOGIA}

Trata-se de um estudo transversal observacional e analítico. $\mathrm{O}$ estudo foi realizado no domicílio dos participantes residentes da cidade de Porto Alegre, Rio Grande do Sul. A amostra foi composta por longevos com idade igual ou superior a 90 anos que foram identificados e acompanhados pelo grupo de Atenção Multiprofissional ao Longevo (AMPAL). Foram incluídos longevos que deambulavam de forma independente e seguros, com ou sem o auxílio de dispositivos de marcha; assim como aqueles que compreendiam comandos verbais. Foram excluídos longevos com déficits sensoriais graves que impediam o entendimento e execução dos procedimentos, assim como, longevos com sequelas motoras importan- 
tes de membros inferiores causadas por Acidente Vascular Cerebral (AVC). Os longevos com dor importante de membros inferiores ou coluna também foram excluídos da pesquisa. Foram avaliadas as variáveis sociodemográficas (sexo, idade, escolaridade e renda mensal) e clínicas (cognição, depressão, número de doenças e número de medicamentos). Os longevos foram questionados quanto ao histórico de queda nos últimos seis meses (resposta "sim" ou "não") e quanto ao medo de cair, utilizando a Falls Efficacy Scale - International - Brasil (FES-I), sendo sem risco quando $<23$ pontos, risco de queda esporádica quanto $\geq 23$ pontos e risco de queda recorrente $\geq$ 31 pontos. Foram avaliados os parâmetros temporais e angulares do aplicativo de smartphone Mobility Suite ${ }^{\circledR}$.

Neste estudo, usamos duas avaliações, o Mini Exame do Estado Mental (MEEM) para rastrear déficit cognitivo e a Geriatric Depression Scale (GDS), para rastrear sintomas depressivos. O MEEM é o teste mais comumente empregado para avaliar a função cognitiva. OMEEM tem pontuação máxima de 30 , sendo o escore de 24 pontos a nota de corte mais adequada. O exame é composto por questões de orientação temporal e espacial, assim como memória imediata, atenção e cálculo, evocação e linguagem ${ }^{7}$. Seguimos pontos de corte distintos conforme o nível educacional, sendo: analfabetos $\geq$ 13; 1 a 8 anos escolaridade $\geq 18$ e $>8$ anos escolaridade $\geq 24^{8}$. Já a GDS utilizada foi a GDS-5. É uma escala composta por cinco perguntas com respostas objetivas a respeito de como o idoso tem se sentido durante a última semana. $\mathrm{O}$ ponto de corte considerado é $\geq 2$ respostas afirmativas?.

Neste estudo, como avaliação principal, foi realizado o TUG. Trata-se de um teste que avalia o desempenho da mobilidade por meio da velocidade ao realizar uma tarefa. $\mathrm{O}$ teste avalia pequenas tarefas motoras essenciais para uma vida independente como o autocontrole postural e o equilíbrio. O teste consiste em levantar-se de uma cadeira com encosto, andar a uma distância de 03 metros, dar a volta e retornar. O tempo gasto para realizar o teste gera uma classificação do risco, sendo risco baixo ( $<10$ segundos), risco médio (10 - 20 segundos) e risco elevado ( $>20$ segundos) $)^{10,11}$.

A realização do TUG se deu pela utilização de um aplicativo de smartphone proposto por profissio- nais da informática ${ }^{6}$. Assim, cada longevo realizou o TUG utilizando um smartphone com sistema operacional Android que foi colocado em uma cinta elástica com velcro e preso no peito, na altura do esterno. Durante o teste, o smartphone executa um aplicativo que utiliza os sensores do giroscópio e acelerômetros do aparelho para extrair parâmetros que quantificam as fases individuais do teste, registrando dessa forma: a duração total do TUG em segundos; a duração da transição de sentado para em pé em segundos; a duração da transição de pé para sentado em segundos; a variação máxima do ângulo do tronco na fase de inclinação para frente em graus e; a velocidade angular máxima durante a fase de inclinação para frente em graus/segundos. Neste aplicativo um feedback é fornecido instantaneamente para o usuário em forma de relatório na tela do smartphone.

Compararam-se as médias (t de Student) e frequências (Qui quadrado) das variáveis sociodemográficas e clínicas entre caidores e não caidores. Os parâmetros do smartphone foram comparados entre caidores e não caidores (t de Student). A regressão logística foi utilizada para avaliar o risco de um longevo ter caído nos últimos seis meses conforme o desempenho de parâmetros significativos do aplicativo na análise univariada. Modelos de regressão logística múltipla foram testados incluindo as variáveis sociodemográficas e clínicas que se mostraram significativos na análise descritiva. Desta forma, foi possível avaliar a chance de o longevo ter relatado queda, atribuído a parâmetros do aplicativo que foram significativos mesmo controlando por variáveis de confusão. Os graus de significância menores que 5\% foram considerados estatisticamente significativos e os entre 5 e $10 \%$ como indicativos de significância. Os dados foram analisados no programa Epi Info ${ }^{\mathrm{TM}} 7.212$

Ainda assim, para avaliar a eficiência do teste na identificação do evento queda foram utilizados parâmetros de sensibilidade e especificidade. Diversos pontos de corte foram utilizados para gerar um gráfico representando uma curva denominada de Receiver Operated Characteristic (ROC).

Esse estudo foi submetido à apreciação do Comitê de Ética em Pesquisa (CEP) da PUCRS sob parecer $n^{\circ}$ 1.772.961. Os idosos incluídos no estudo autorizaram a sua participação pela assinatura do Termo de Consentimento Livre e Esclarecido (TCLE). 


\section{RESULTADOS}

O estudo contou com a participação de avaliação de 98 longevos. Na Tabela 1 são apresentadas as diferenças nas características sociodemográficas e clínicas entre longevos caidores e não caidores. Dos avaliados, 26,5\% referiram queda pelo menos uma vez nos últimos seis meses. Na análise das variáveis demográficas, o percentual de mulheres referindo quedas foi menor $(25,76 \%)$, no entanto não significativo $(\mathrm{p}=0,492)$. A média de idade dos longevos caidores foi de 92 $\pm 3,14$ anos, mostrando-se um pouco superior à dos não caidores $(\mathrm{p}=0,636)$. Os longevos com quatro a oito anos de estudo foram os que referiram, proporcionalmente, mais quedas $(31,82 \%$, $\mathrm{p}=0,294)$, o mesmo observado nos com renda média entre $\mathrm{R} \$ 5,1$ a $\mathrm{R} \$ 10$ mil $(27,27 \%)$, embora não significativo $(p=0,334)$.

$\mathrm{Na}$ análise das variáveis clínicas, em relação ao MEEM, 26,76\% dos longevos que tinham o exame normal referiram quedas, embora não significativo $(\mathrm{p}=0,575)$. Já em relação à GDS, a média de sintomas depressivos foi significativamente $(p=0,001)$ maior nos longevos com história de queda. Proporcionalmente, 46,34\% dos longevos que apresentaram dois ou mais sintomas depressivos referiram quedas $(\mathrm{p}<0,001)$. Dos longevos que apresentavam mais de uma doença crônica (multimorbidade) associada, 26,83\% deles referiram quedas, o que não foi considerado significativo $(p=0,575)$. Entre os longevos fazendo uso de cinco ou mais medicamentos (polifarmácia), $28,79 \%$ deles referiram queda $(\mathrm{p}=0,318)$, proporção essa também maior que o esperado $(27,27 \%)$ entre os que apresentavam medo de cair sempre ou às vezes $(\mathrm{p}=0,518)$, embora ambos não significativos.

Na Tabela 2 é apresentada a distribuição dos longevos caidores e não caidores na classificação do TUG e as diferenças médias dos seus parâmetros avaliados pelo aplicativo Mobility Suite ${ }^{\circledR}$. Na análise do tempo avaliado pelo TUG, os caidores apresentaram uma média de 20,8 $\pm 8,94$ segundos $(p=0,800)$, sendo essa média de 0,6 segundos maior que os não caidores, porém não significativo. Os longevos classificados com risco de queda alto, pela classificação do $T U G$, apresentaram proporção maior de quedas $(32,50 \%)$ em relação aos com risco médio (20,37\%), não sendo significativo $(\mathrm{p}=0,233)$. Na análise dos parâmetros avaliados pelo aplicativo Mobility Suite ${ }^{\circledR}$, longevos caidores apresentaram maiores valores na fase de pé para sentado e na fase de variação do ângulo. Por outro lado, apresentaram menores valores na fase velocidade do ângulo e na fase de pé para sentado, embora nenhum parâmetro tenha se mostrado significativo quando comparado os dois grupos. Identificou-se também, na Tabela 2, que o parâmetro que apresentou maior diferença percentual entre longevos caidores e não caidores foi a fase de sentado para em pé (15\%).

$\mathrm{Na}$ Tabela 3 são apresentadas as razões de chance para o relato de queda nos últimos seis meses nos modelos univariados e completo. A análise de regressão logística foi utilizada para verificar se a presença significativamente maior de sintomas depressivos entre os longevos com história de queda estaria relacionada ou dependente de outro(s) fator (es) estudados. Para tanto foram criados modelos de regressão logística univariados, com um modelo para cada uma das variáveis na avaliação da chance maior ou menor do longevo apresentar história de queda, e completo com todas as variáveis estudadas. A única variável significativa tanto nos modelos univariados quanto no modelo completo foi a GDS, demonstrando que longevos com dois ou mais sintomas depressivos apresentam maior chance de história de queda. 
Tabela 1. Diferenças nas características sociodemográficas e clínicas entre longevos caidores e não caidores

\begin{tabular}{|c|c|c|c|}
\hline Variáveis sociodemográficas & Não caidores & Caidores & $\mathbf{p}$ \\
\hline \multicolumn{4}{|l|}{ Sexo } \\
\hline Feminino & $49(74,24 \%)$ & $17(25,76 \%)$ & 0,492 \\
\hline Masculino & $23(71,88 \%)$ & $9(28,13 \%)$ & \\
\hline Idade & $91,88 \pm 2,65$ & $92,19 \pm 3,14$ & 0,636 \\
\hline \multicolumn{4}{|l|}{ Escolaridade } \\
\hline Analfabeto & $5(83,33 \%)$ & $1(16,67 \%)$ & 0,294 \\
\hline 1 a 3 anos & $9(81,82 \%)$ & $2(18,18 \%)$ & \\
\hline 4 a 8 anos & $30(68,18 \%)$ & $14(31,82 \%)$ & \\
\hline$>8$ anos & $28(75,68 \%)$ & $9(24,32 \%)$ & \\
\hline Renda (média $\pm \mathrm{DP}$ ) & $4009,7 \pm 4561,88$ & $3692,3 \pm 3357,37$ & 0,746 \\
\hline $\mathrm{R} \$ 1,0$ a $5,0 \mathrm{mil}$ & $57(73,08 \%)$ & $21(26,92 \%)$ & 0,334 \\
\hline $\mathrm{R} \$ 5,1 \mathrm{a} 10 \mathrm{mil}$ & $8(72,73 \%)$ & $3(27,27 \%)$ & \\
\hline $\mathrm{R} \$ \geq 10,1 \mathrm{mil}$ & $7(77,78 \%)$ & $2(22,22 \%)$ & \\
\hline \multicolumn{4}{|l|}{ Variáveis Clínicas } \\
\hline MEEM (média $\pm D P$ ) & $21,31 \pm 5,34$ & $21,50 \pm 5,25$ & 0,882 \\
\hline Alterado & $20(74,07 \%)$ & $7(25,93 \%)$ & 0,575 \\
\hline Normal & $52(73,24 \%)$ & $19(26,76 \%)$ & \\
\hline GDS (média \pm DP) & $1,27 \pm 1,02$ & $2,03 \pm 0,87$ & $0,001 *$ \\
\hline Alterado ( $\geq 2$ sintomas) & $22(53,66 \%)$ & $19(46,34 \%)$ & $<0,001^{*}$ \\
\hline Normal $(<2$ sintomas & $50(87,72 \%)$ & $7(12,28 \%)$ & \\
\hline Morbidades (média $\pm \mathrm{DP}$ ) & $4,16 \pm 1,80$ & $4,53 \pm 1,90$ & 0,377 \\
\hline Com multimorbidade( $\geq$ 2doenças) & $60(73,17 \%)$ & $22(26,83 \%)$ & 0,575 \\
\hline Sem multimorbidade (<2 doenças) & $12(75,00 \%)$ & $4(25,00 \%)$ & \\
\hline Medicamentos (média $\pm \mathrm{DP}$ ) & $5,50 \pm 2,58$ & $6,23 \pm 2,53$ & 0,217 \\
\hline Com polifarmácia ( $\geq$ 5medicamentos) & $47(71,21 \%)$ & $19(28,79 \%)$ & 0,318 \\
\hline Sem polifarmácia ( $<5$ medicamentos) & $25(78,13 \%)$ & $7(21,88 \%)$ & \\
\hline \multicolumn{4}{|l|}{ Medo de cair } \\
\hline Às vezes/Sempre & $40(72,73 \%)$ & $15(27,27 \%)$ & 0,518 \\
\hline Nunca & $32(74,42 \%)$ & $11(25,58 \%)$ & \\
\hline Total & $72(73,5 \%)$ & $26(26,5 \%)$ & \\
\hline
\end{tabular}

$\mathrm{DP}=$ Desvio-padrão; MEEM= Mini Exame do Estado Mental; GDS=Geriatric Depressive Scale; * $\mathrm{p}<0,05$. 
Tabela 2. Distribuição dos longevos caidores e não caidores na classificação do TUG e as diferenças médias dos seus parâmetros avaliados pelo aplicativo UAH Mobility Suite ${ }^{\circledR}$

\begin{tabular}{lcccc}
\hline & Não caidores & Caidores & $\%$ & p \\
\hline TUG (s) (média \pm DP) & $20,2 \pm 9,28$ & $20,8 \pm 8,94$ & & 0,800 \\
Baixo & $2(50,00 \%)$ & $2(50,00 \%)$ & & 0,233 \\
Médio & $43(79,63 \%)$ & $11(20,37 \%)$ & & \\
Alto & $27(67,50 \%)$ & $13(32,50 \%)$ & & \\
Parâmetros TUG (média \pm DP) & & & & \\
Sentado para em pé (s) & $1,67 \pm 0,69$ & $1,76 \pm 0,56$ & $15 \%$ & 0,551 \\
Pé para sentado (s) & $2,07 \pm 1,02$ & $1,97 \pm 0,82$ & $5 \%$ & 0,641 \\
Variação do ângulo $(\mathrm{g})$ & $43,13 \pm 14,47$ & $44,08 \pm 10,81$ & $2 \%$ & 0,762 \\
Velocidade do ângulo $(\mathrm{g} / \mathrm{s})$ & $94,65 \pm 36,51$ & $92,14 \pm 31,96$ & $3 \%$ & 0,757 \\
\hline
\end{tabular}

$\mathrm{DP}=$ Desvio-padrão; ${ }^{*} \mathrm{p}<0,05$.

Tabela 3. Razões de chance para o relato de queda nos últimos seis meses, modelos univariados e completo.

Variável

Modelos Univariados RC ( \pm IC) $\quad$ Modelo Completo RC $( \pm$ IC)

\begin{tabular}{llc}
\hline Sexo (ref. feminino) & & \\
Masculino & $1,13(0,44 \pm 2,91)$ & $2,24(0,60 \pm 8,34)$ \\
Idade (anos) & $1,02(0,89 \pm 1,22)$ & $1,08(0,90 \pm 1,30)$ \\
\hline Escolaridade (ref. >8 anos) & & \\
\hline Analfabeto/> 8 anos & $0,62(0,06 \pm 6,05)$ & $0,50(0,03 \pm 8,19)$ \\
1 a 3 anos/> 8 anos & $0,70(0,12 \pm 3,81)$ & $0,50(0,06 \pm 3,90)$ \\
$\quad \begin{array}{l}\text { a } 8 \text { anos/> } 8 \text { anos } \\
\text { Renda (ref. até R\$5,0 mil) }\end{array}$ & $1,45(0,54 \pm 3,88)$ & $1,39(0,43 \pm 4,52)$ \\
\hline Entre 5,1 e 10.0/Até 5,0 & & $0,71(0,11 \pm 4,63)$ \\
Superior a 10,1/Até 5,0 & $1,01(0,24 \pm 4,20)$ & $0,71(0,10 \pm 5,01)$ \\
MEEM (pontos) & $0,77(0,15 \pm 4,03)$ & $0,86(0,24 \pm 3,10)$ \\
GDS (ref. $\leq \mathbf{2}$ sintomas) & $1,00(0,40 \pm 2,90)$ & $9,4(2,81 \pm 31,67) *$ \\
Morbidades (no doenças) & $6,2(2,27 \pm 16,8)^{*}$ & $0,72(0,16 \pm 3,20)$ \\
Medicamentos (no ${ }^{\mathbf{0}}$ medicamentos) & $0,91(0,26 \pm 3,12)$ & $0,85(0,23 \pm 3,07)$ \\
Medo de cair (ref. Não) & $0,70(0,25 \pm 1,87)$ & $0,78(0,22 \pm 2,76)$ \\
TUG (s) & $1,09(0,44 \pm 2,70)$ & $1,12(0,34 \pm 3,70)$ \\
Sentado para em pé (s) & $1,27(0,57 \pm 2,86)$ & $0,83(0,25 \pm 2,77)$ \\
Pé para sentado (s) & $1,14(0,59 \pm 2,22)$ & $0,69(0,33 \pm 1,43)$ \\
Variação do ângulo (g) & $0,89(0,54 \pm 1,45)$ & $1,00(0,95 \pm 1,06)$ \\
Velocidade do ângulo (g/s) & $1,00(0,97 \pm 1,04)$ & $1,00(1,00 \pm 1,02)$ \\
\hline
\end{tabular}

ref=valor de referência; $\mathrm{RC}=$ Razão de chance; $\mathrm{IC}=$ Intervalo de confiança de $95 \%$; $\mathrm{p}<0,05$. 
O gráfico dos parâmetros representa uma Curva ROC (Receiver Operated Characteristic) e a linha diagonal a relação sensibilidade/especificidade que classifica o teste como sendo válido. Quanto mais afastada dessa linha a curva de ROC for (mais próximo do ângulo formado pela sensibilidade de $100 \%$ e especificidade de 100\%) mais eficaz é o teste e maior a área abaixo da linha.

No caso dos resultados do teste completo do TUG (Figura 1.A), observou-se que o tempo que apresenta melhor relação sensibilidade/especificidade foi o de 19,81 segundos, com $54 \%$ de sensibilidade e $61 \%$ para especificidade. Tempo superior a este indicaria risco de queda. A linha ROC ficou muito próxima à linha diagonal, demonstrando ser um parâmetro pouco eficaz na predição do relato de queda. Na fase de sentado para em pé (Figura 2.A), observou-se que a melhor relação entre os dois parâmetros foi a que apresentava uma sensibilidade de $50 \%$ e especificidade de $61 \%$, em que o tempo foi de 1,68 segundos. Tempo superior a este indicaria risco de queda. A linha ROC ficou mais afastada da linha diagonal que nos permite dizer que esta fase do TUG possa ser mais eficaz na predição do relato de queda do que o teste completo do TUG. Na fase de pé para sentado (Figura 1.C), observou-se que a melhor relação entre os dois parâmetros foi a que apresentava uma sensibilidade de $50 \%$ e especificidade de $53 \%$, em que o tempo foi de 1,91 segundos. Tempo inferior a este indicaria risco de queda. A linha ROC ficou em ambos os lados da linha diagonal demonstrando ser pouco eficaz. Em relação à variação do ângulo (Figura 1.D), observou-se que a melhor relação entre os dois parâmetros foi a que apresentava uma sensibilidade de $50 \%$ e especificidade de $58 \%$, em que o ângulo foi de 42,2 graus. Ângulo superior a este indicaria risco de queda. A linha ROC ficou muito mais próxima da linha diagonal do que os outros parâmetros do TUG demonstrando também ser pouco eficaz. Em relação à velocidade do ângulo (Figura 1.E), observou-se que a melhor relação entre os dois parâmetros foi a que apresentava uma sensibilidade de $50 \%$ e especificidade de $58 \%$, em que a velocidade foi de 83,74 graus/segundos. Velocidade inferior a esta indicaria risco de queda. A linha ROC ficou um pouco mais afastada da linha diagonal do que a fase de pé para sentado demonstrando ser mais eficaz do que essa fase, mas menos eficaz do que a fase de sentado para em pé.
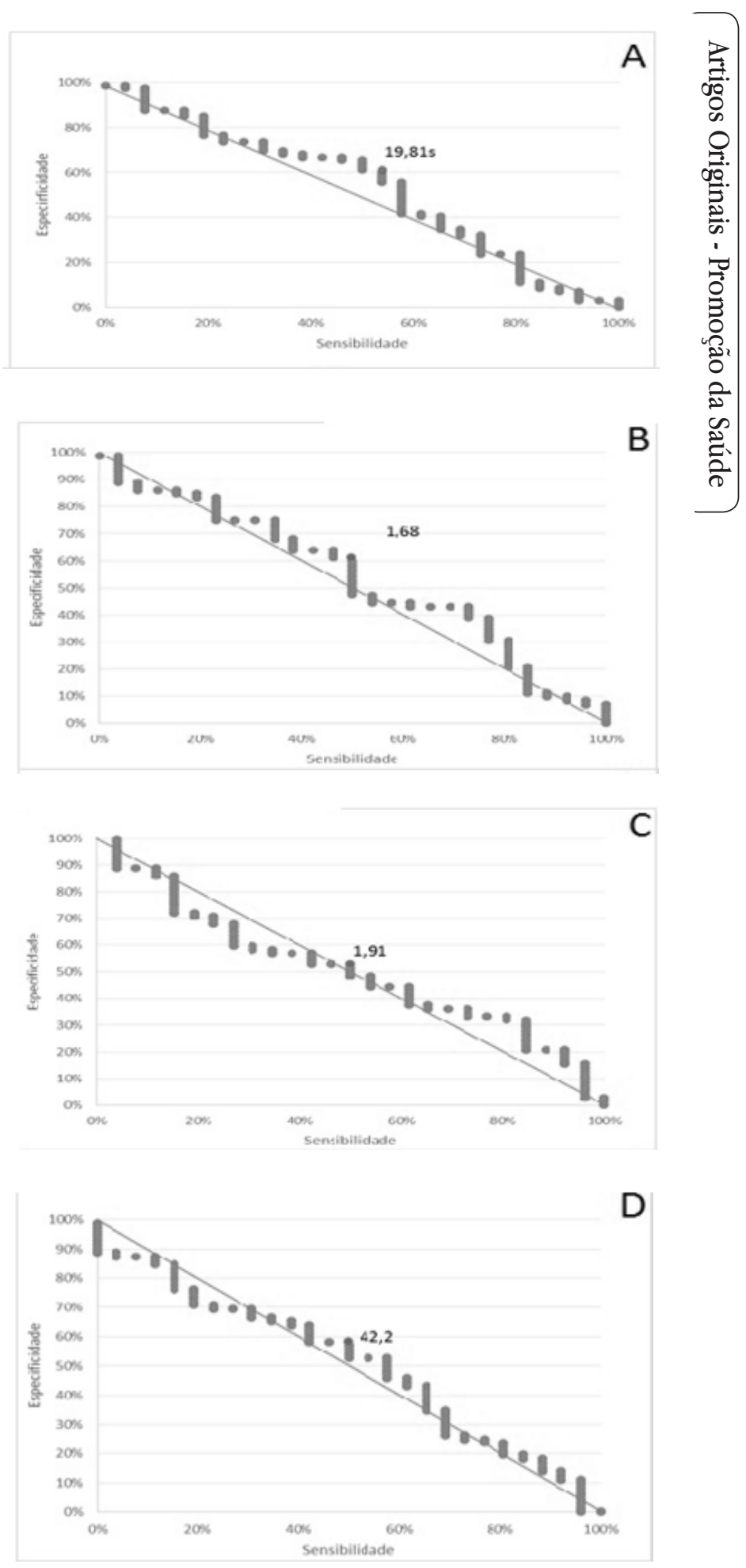


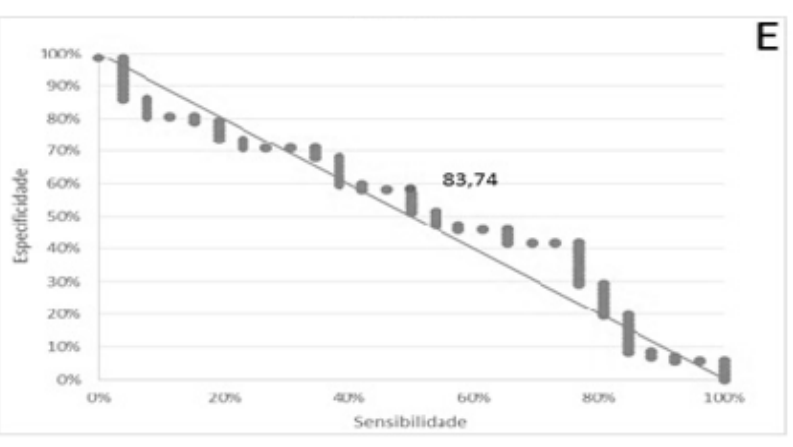

Figura 1. Sensibilidade e especificidade para o relato de queda nos testes: TUG completo (A), fase sentado para em pé (B), fase em pé para sentado $(C)$, variação do ângulo (D) e velocidade do ângulo (E)

\section{DISCUSSÃO}

A literatura aponta que a prevalência de quedas aumenta com a idade, chegando a $50 \%$ em longevos ${ }^{2}$. Neste estudo, a prevalência mostrou-se abaixo do esperado (26,5\%). No entanto, apresentou-se semelhante à pesquisa de Brito et al. ${ }^{13}$ que, ao estudarem a associação entre quedas e capacidade funcional em longevos, encontraram uma prevalência de $27,7 \%$. Acredita-se que tal resultado deva-se ao fato de que grande parte da população longeva avaliada em nosso estudo encontrava-se funcionalmente ativa e em bom estado geral de saúde.

Observou-se que o percentual de quedas foi maior nos homens, fato que contraria a maioria dos achados. Estudos apresentaram maior prevalência de quedas entre mulheres, sugerindo como causas a maior fragilidade das mesmas em relação aos homens, assim como maior prevalência de doenças crônicas e uma maior exposição a atividades domésticas, que geram um comportamento de maior risco ${ }^{14}$. O fato de termos observado mais quedas nos homens, nos leva a crer que tenha sido um resultado do acaso, pois não observamos diferença estatisticamente significativa. Por outro lado, nos faz refletir que possa ser que os homens apresentem fator latente, não avaliado, que pudesse ser contribuinte para a queda.

A frequência de quedas mostrou-se superior entre os mais velhos. Tal achado corrobora com Rolita et al. ${ }^{15}$ que afirmam que o nível de fragilidade de idosos aumenta com o passar dos anos, ao mesmo passo que o nível de funcionalidade diminui, favorecendo maior proporção a quedas.

Neste estudo, longevos com renda média referiram mais quedas. Tal achado contraria Abreu et al. ${ }^{16}$ que observaram risco aumentado de queda em idosos de baixa renda. Os mesmos apontam que baixos salários, apresentam-se como um fator contribuinte para dificuldades enfrentadas no domicílio desses idosos, ao passo que os fazem enfrentar maiores riscos decorrentes de um ambiente prejudicial. Da mesma forma, longevos com escolaridade média referiram mais quedas. Tal achado contraria o estudo de Abreu et al. ${ }^{17}$ que observaram uma associação entre baixa escolaridade e queda. Uma possível explicação é que idosos com baixos níveis de instrução se preocupam menos com cuidados de saúde. Adicionalmente, o nível educacional influencia a percepção espacial dos idosos. A não observância da relação entre baixa renda e escolaridade com o risco de queda, confirma a ausência de significância em nosso estudo. Assim, acredita-se que um maior percentual de quedas foi observado nos longevos com renda e escolaridade média pela representação da amostra, ao passo que tivemos que excluir, áreas consideradas de risco, a fim de preservar a segurança dos profissionais. Dessas áreas de risco excluídas todas abrangiam regióes de comunidades de baixa renda, levando-nos a crer que abrangiam também uma população de baixa escolaridade.

Diante do déficit cognitivo, observou-se que os longevos que não apresentaram alteração referiram mais quedas. Corroborando com nossos achados uma hipótese seria que idosos sem déficit cognitivo são mais ativos, sendo expostos a alguns riscos, principalmente os externos à residência ${ }^{18}$. Por outro lado, contrariando nossa pesquisa, para Aveiro et al. ${ }^{19}$, idosos com déficit cognitivo estão mais susceptíveis a sofrer quedas, ao passo que apresentam respostas protetoras comprometidas. A não significância dessa relação e as duas hipóteses apontadas pelos autores fala a favor da necessidade de confirmação em estudos futuros.

A depressão mostrou-se a única variável significativa em nosso estudo, demonstrando que longevos com dois ou mais sintomas depressivos apresentam maior chance de história de queda. Para Matias et al. ${ }^{20}$, situações vivenciadas pelos idosos, como viuvez, solidão, dependência e doenças, podem contribuir para desestruturações psíquicas que condicionam o quadro de depressão, causando importantes danos à saúde. A depressão não diagnosticada e não tratada piora a condição funcional 
e aumenta o risco para outras doenças, assim como para episódios de quedas ${ }^{20}$. Em relação à queda, parece estar atribuída à diminuição de neurotransmissores a nível sináptico, provocando o retardamento de reações, que, associadas às alterações próprias do envelhecimento, potencializam ainda mais o risco ${ }^{20}$. O desenho deste estudo não permite afirmar o fator causal desta relação, depressão sendo a causa da queda ou queda sendo a causa da depressão. Porém, a literatura nos leva a crer que a depressão é fator causador de quedas, principalmente pela ingestão de medicamentos antidepressivos ou, ainda, por diversas outras razões como, por exemplo, alterações provocadas pela depressão que podem ocasionar indiferença ao meio ambiente, como alteração do nível da atenção e redução do comprimento da passada ${ }^{21}$.

No presente estudo, longevos com um número maior de doenças crônicas referiram mais quedas. Para Rodrigues et al. ${ }^{22}$ o número de doenças, assim como outros problemas de saúde contribuem para o declínio da funcionalidade e aumento da fraqueza muscular, que associados às limitações de mobilidade podem acarretar maior predisposição às quedas.

Nesta pesquisa, longevos que utilizavam maior número de medicamentos referiram mais quedas. Para Abreu et al. ${ }^{17}$, a utilização de cinco ou mais medicamentos apresenta uma associação com a ocorrência de queda. A polifarmácia em idosos pode causar mais frequentemente quedas, pois aumenta a incidência de efeitos colaterais e interações medicamentosas fazendo com que os pacientes apresentem respostas mais lentas à perda do equilíbrio.

Em relação ao medo de cair, observou-se que longevos que apresentavam medo de cair às vezes ou sempre referiram mais quedas. Para Malini et al. ${ }^{23}$, apesar de existirem circunstâncias nas quais o medo de queda possa ser considerado um fator protetor, quando, por exemplo, este impede que o indivíduo se arrisque em situações que poderiam provocar quedas, em outras, este medo pode ser exagerado ou desproporcional em relação ao estímulo, causando restrições físicas e até mesmo a queda.

$\mathrm{Na}$ análise do tempo para realizar o teste completo do $T U G$, os caidores levaram mais tempo do que os não caidores. Não encontramos na literatura estudos que avaliaram o desempenho de longevos caidores e não caidores através do TUG, sendo então um resultado inédito na literatura científica. No entanto, Lusardi et al. ${ }^{24}$ avaliaram idosos por década de idade, a fim de determinar valores de referência de sete testes funcionais, sendo um deles o TUG. Em nonagenários a média do tempo total de realização do teste foi de 17,7 segundos, valor menor do que o observado no presente trabalho. Embora ambos os estudos tenham avaliado nonagenários, a principal diferença nos longevos estudados por Lusardi et al. ${ }^{24}$, que consequentemente apresentaram um valor mais satisfatório no TUG, deve-se ao fato dos participantes terem realizado os testes em um centro comunitário, o que de certa forma nos leva a crer que eles tenham utilizado uma amostra com condições funcionais superiores aos dos longevos avaliados pelo AMPAL. Em relação à classificação de risco do TUG proposta por Podsiadlo e Richardson ${ }^{10}$, os longevos classificados com alto risco de queda, apresentaram uma proporção maior de quedas em relação aos com médio risco. Não encontramos na literatura estudos que tenham avaliado especificamente o risco de quedas de longevos através do $T U G$, seguindo alguma classificação de risco. No entanto, Queiroz et al. ${ }^{25}$ estudaram o risco de quedas, através do TUG e utilizando a classificação de Podsiadlo e Richardson ${ }^{13}$, em idosos com média de idade de 70 anos, encontraram resultados que apoiam nossos achados. Dos idosos classificados em alto risco de queda, $28,6 \%$ eram longevos e desses $42,9 \%$ tinham histórico de queda, observando uma relação diretamente proporcional entre longevidade e prevalência de quedas.

A fase de levantar da cadeira engloba ações e estabilizações musculares de coluna, mas principalmente de membros inferiores ${ }^{26}$. Neste estudo, longevos caidores demoraram mais tempo para levantar-se. Fase com maior diferença entre os grupos. Pelicioni et al. ${ }^{26} \mathrm{com}$ pararam as fases de levantar e andar de jovens e idosos e observaram, que esta é a fase que sofre maior influência do processo de envelhecimento, favorecendo o risco de queda. Isso se deve à menor produção de força e menor velocidade de extensão de membros inferiores observada com o passar dos anos. Tal achado nos leva a crer que se quanto maior a idade, pior é o desempenho nesta fase $\mathrm{e}$ maior será o risco de queda, justificando assim, a maior 
alteração em longevos caidores.

Neste estudo, longevos caidores apresentaram um tempo menor na fase de pé para sentado, achado este, que, se levando em consideração o tempo total de realização do teste, mostra-se satisfatório. No entanto, defendemos a hipótese de que, neste caso, em longevos caidores, apresentar um tempo menor nesta fase mostra-se sugestivo de maior risco de queda. Tal argumento baseia-se no estudo de Fonseca e Scheicher ${ }^{27}$ que, ao avaliarem a postura estática de idosos, verificaram que a projeção anterior, ou seja, um deslocamento do centro de massa anteriormente; foi indicativo de aumento do desequilíbrio em idosos. Para eles, durante o processo de envelhecimento, aumentam-se as assimetrias posturais que podem afetar o mecanismo de controle de equilíbrio, levando às quedas. Este deslocamento de centro de massa, observado por estes autores, também pode justificar a maior variação de ângulo observada em longevos caidores de nosso estudo. Já do ponto de vista muscular, para Pelicioni et al. ${ }^{26}$, as alterações musculoesqueléticas e desafios impostos pelo declínio do controle postural, interferem no desempenho de sentar-se ou levantar-se de uma cadeira. Desta forma, a fraqueza de músculos dos membros inferiores, podem contribuir para que os longevos caidores, não controlem bem a descida nesta fase. Assim, podemos inferir, tanto com os resultados observados na presente pesquisa, quanto por essa base teórica que a maior velocidade de se sentar está diretamente relacionada ao pior desempenho na fase. Dessa forma, longevos com maior velocidade para sentar teriam maior risco de queda.

Neste estudo observamos que caidores apresentaram menor velocidade do ângulo quando comparados aos não caidores. Para Cordeiro et al. ${ }^{28}$, um acometimento comum com o envelhecimento é a perda de mobilidade da coluna vertebral, ao passo que exige manutenção dos níveis de flexibilidade. A manutenção dessa mobilidade é necessária, pois pode interferir consequentemente na velocidade de ação de certos movimentos aumentando as chances de quedas. Assim, acredita-se que a menor velocidade de ângulo apresentada pelos longevos caidores possa ser indicativa de um comprometimento de mobilidade da coluna vertebral, ocasionando um pior desempenho no teste.
É a primeira vez na literatura científica que se avalia o desempenho de longevos caidores e não caidores, através do teste TUG, quantificando as suas fases. Embora não tenhamos encontrado uma sensibilidade $\mathrm{e}$ especificidade satisfatórias, os parâmetros encontrados são importantes para comparações futuras a fim de se comprovar ou rejeitar os achados apontados.

Em relação ao teste completo do TUG observou-se, em nosso estudo, que um tempo superior a 19,81 segundos indicaria risco de queda em longevos. Achado este que através da classificação de Podsiadlo e Richardson ${ }^{10}$ para idosos jovens, enquadrou-se na transição do risco médio (10 - 20 segundos) para o risco alto de quedas ( $>20$ segundos). Mais longevos não caidores apresentaram risco médio de queda (60\%) quando comparado aos não caidores (43\%). Para risco alto de queda, $37,5 \%$ dos não caidores e $50 \%$ dos caidores estariam nesse critério. Assim, concluímos que os pontos de corte propostos por Podsiadlo e Richardson ${ }^{10}$ não são adequados para a população longeva, principalmente a classificação de risco médio.

Dessa forma, baseados em nossos achados, questionamos a confiabilidade do $T U G$ na predição do risco de quedas em idosos. Barry et al. ${ }^{29}$ realizaram uma meta-análise a fim de determinar o valor preditivo do teste TUG para o evento queda. Em seus achados, observaram que o TUG não se mostrou ser um preditor significativo de quedas, pois apresentou uma capacidade limitada, não sendo indicada a sua utilização de forma isolada. O TUG é um teste que avalia um único parâmetro temporal, mas é composto por três fases distintas (levantar, caminhar e sentar), que podem influenciar de forma isolada ou conjunta o risco de quedas. Assim, apoiando a inovação proposta por nosso estudo, Greene et al. ${ }^{30}$, ao quantificarem as fases do TUG através de sensores corporais em um laboratório de movimento, aumentaram a precisão de quedas, para quase $80 \%$, sugerindo, assim, que a avaliação das suas fases mostra-se como um método inovador e promissor para avaliar o futuro risco de quedas em idosos.

Em relação aos parâmetros avaliados pelo aplicativo, podemos destacar alguns pontos de corte, em cada fase distinta do teste, que foram considerados indicativos de risco de queda, sendo eles: fase de sentado para em 
pé, >1,68; fase de pé para sentado, <1,91 segundos; variação do ângulo, $>42,2$ graus; e velocidade do ângulo, $<83,74$ graus / segundos indicaria risco de queda.

Neste estudo utilizamos do histórico de queda como parâmetro de risco para a ocorrência de um evento futuro. Sendo assim, dois fatores podem ter interferido em nossas análises. Primeiro, a queda ocorreu antes da realização do TUG; sendo assim, o medo de cair pode ter interferido no desempenho do teste. Segundo, a causa da queda pode não ter ocorrido exclusivamente por fatores intrínsecos, mas preferencialmente ou em associação a fatores extrínsecos. Desta forma, para se avaliar o papel desta avaliação na predição de quedas, o ideal seria comparar o seu desempenho com um teste específico de equilíbrio. Assim, apoia-se o surgimento de novas pesquisas a fim de complementar esta avaliação, no entanto, utilizando-se de um acompanhamento longitudinal a fim de confirmar ou rejeitar o papel do TUG no rastreio de futuros eventos.

O presente trabalho teve como resultado mais expressivo a relação entre depressão e o relato de queda. $\mathrm{O}$ desenho transversal da presente pesquisa associado à avaliação da presença de sintomas depressivos apenas após a queda não nos disponibiliza a interpretação causal dos resultados, não permitindo identificar se a depressão é uma causa ou consequência da queda.

Por outro lado, tratando-se de um estudo inovador, que se utilizou um software mobile, visualizamos outros dois pontos técnicos que podem ser considerados limitantes. O primeiro está relacionado aos recursos físicos do smartphone, em que não foi determinada com exatidão a acurácia dos parâmetros rastreados e mapeados através do giroscópio e acelerômetros disponíveis e; o segundo está relacionado ao software, que pode ter apresentado restrições lógicas de programação durante a execução do teste.

\section{CONCLUSÃO}

Conclui-se que o aplicativo de smartphone Mobility Suite ${ }^{\circledR}$ foi capaz de avaliar o desempenho de longevos caidores e não caidores, embora nenhum parâmetro tenha sido significativo quando comparados os grupos. Na identificação de quais parâmetros apresenta- ram mais similaridades e disparidades, longevos caidores obtiveram menores valores em dois componentes (fase de pé para sentado e velocidade do ângulo) e obtiveram maiores valores nos outros dois componentes (fase de sentado para em pé e variação do ângulo). Assim, de forma geral, em todas as fases do teste, longevos caidores apresentaram resultados distintos de longevos não caidores, que baseados na literatura, se mostraram justificáveis para favorecer a ocorrência de queda. Assim, através deste estudo, obtiveram-se parâmetros iniciais sobre a quantificação do TUG em longevos, possibilitando o surgimento de novas pesquisas a fim de implementar pontos de corte adequados para a população longeva.

Em relação às variáveis sociodemográficas $\mathrm{e}$ clínicas, a depressão mostrou-se a única estatisticamente significativa, indicando que longevos com sintomas depressivos apresentam mais chances de cair. No entanto, esta relação ainda necessita de outros estudos, a fim de confirmar a sua relação causal.

\section{AGRADECIMENTOS}

À Coordenação de Aperfeiçoamento de Pessoal de Nível Superior - Brasil (Capes) - Código de Financiamento 001 pelo apoio financeiro na realização desta pesquisa.

\section{REFERÊNCIAS}

1. Porciúncula RCR, Carvalho EF, Barreto KML, Leite, VMM. Perfil socioepidemiológico e autonomia de longevos em Recife-PE, Nordeste do Brasil. Rev Bras Geriatr Gerontol. 2014;17(2):315-25.

2. Guerra HS, Alves e Sousa R, Bernardes DCF, Santana JA, Barreira LM. Prevalência de quedas em idosos na comunidade Revista Saúde e Pesquisa. 2016;9(3):547-55.

3. Gomes ECC, Marques APO, Leal MCC, Barros BP. Fatores associados ao risco de quedas em idosos institucionalizados: uma revisão integrativa. Ciência \& Saúde Coletiva. 2014;19(8):3543-51.

4. Carvalho LFS. Utilização de Dispositivos Móveis na aprendizagem da Matemática no $3^{\circ}$ Ciclo. [dissertação]. Porto: Universidade Portucalense Infante D. Henrique; 2015. 153 p. 
5. GuzmánJC, SilvaRG,Guzmán-venegas R. Reproducibilidad de los tiempos de ejecución de la prueba de Timed Up and Go, medidos con acelerómetros de smartphonesen personas mayores residentes en la comunidade. Revista Española de Geriatría y Gerontología. 2017; 52(1): 249-52.

6. Milosevic M, Jovanov E, Milenković A. Quantifying Timed-Up-and-Go Test: A Smartphone Implementation. Conference: Body Sensor Networks (BSN), IEEE. 2013.

7. Folstein M, Folstein S, Mchugh P. "Mini-Mental State". A practical method for grading the cognitive state of patients for the clinicians. J Psychiatr Res. 1975;12(1): 189-98.

8. Bertolucci PHF, Brucki SMD, Campacci SR, Julian Y. O Mini Exame do Estado Mental em uma população geral impacto da escolaridade. Arq Neuropsiquiatr. 1994; 52(1):1-7.

9. Almeida MSC. Efetividade da escala de depressão geriátrica de cinco itens em população idosa da comunidade[tese]. Porto Alegre: Pontifícia Universidade Católica do Rio Grande do Sul, Faculdade de Medicina; 2010.137 p.

10. Podsiadlo D, Richardson S. The timed "Up \& Go": a test of basic functional mobility for frail elderly persons. J Am Geriatr Soc.1991;39(2):142-48.

11. Wall JC, Bell C, Campbell S, Davis J. The Timed getup-and-go test revisited: Measurement of the component tasks. J Rehabil Res Dev. 2000;37(1):109-14.

12. Bós AJG. Epi Info ${ }^{\circledR}$ sem Mistérios: Um manual prático. Porto Alegre: EDIPUCRS; 2012. 212 p.

13. Brito TA, Fernandes MH, Coqueiro RS, Jesus CS. Quedas e capacidade funcional em idosos longevos residentes em comunidade. Texto Contexto Enferm. 2013;22(1):43-51.

14. Gasparotto LPR, Falsarella GR, Coimbra AMV. As quedas no cenário da velhice: conceitos básicos e atualidades da pesquisa em saúde. Rev Bras Geriatr Gerontol. 2014;17(1):201-9.

15. Rolita L, Spegman A, Tang X, Cronstein BN. Greater number of narcotic analgesic prescriptions for osteoarthritis is associated with falls and fractures in elderly adults.J Am Geriatr Soc. 2013; 61(3):335-40.

16. Abreu DROM, Azevedo RCS, Silva AMC, Reiners AAO,
Abreu HCA. Fatores associados à recorrência de quedas em uma coorte de idosos. Ciênc Saúde Coletiva. 2016;21(3): 3439-46.

17. Abreu HCA, Reiners AAO, Azevedo RCS, Silva AMC, Abreu DROM, Oliveira AD. Incidência e fatores preditores de quedas de idosos hospitalizados. Rev Saúde Pública. 2015;49(37):1-9.

18. Cruz, DT, Cruz FM, Ribeiro, AL, Veiga CL, Leite ICG. Associação entre capacidade cognitiva e ocorrência de quedas em idosos. Cad Saúde Colet. 2015; 23(4): 386-393.

19. Aveiro MC, Driusso P, Barham EJ, Pavarini SCI, Oishi J. Mobilidade e risco de quedas de população idosa da comunidade de São Carlos. Ciênc Saúde Coletiva. 2012;17(9):2481-88.

20. Matias AGC, Fonseca MA, Matos MAA. Análise fatorial de sintomas depressivos e ocorrência de quedas em idosos. Scientia Medica. 2015; 25(1):1-8.

21. Prata HL, Alves Junior ED, Paula FL, Ferreira SM. Envelhecimento, depressão e quedas: um estudo com os participantes do Projeto Prev-Quedas. Fisioter Mov.2011; 24(3): 437-443.

22. Rodrigues IG, Fraga GP, Barros MBA. Quedas em idosos: fatores associados em estudo de base populacional. Rev Bras Epidemiol. 2014;1(1):705-18.

23. Malini F, Lopes C, Lourenço R. Medo de quedas em idosos: uma revisão da literatura. Revista HUPE. 2014;13(2):38-44.

24. Lusardi MM, Pellecchia GL, Schulman M. Functional Performance in Community Living Older Adults. Journal of Geriatric Physical Therapy. 2003;26(3):1422,2003.

25. Queiroz L, Lira S, Sasaki A. Identificação do risco de quedas pela avaliação da mobilidade funcional em idosos hospitalizados. Revista Baiana de Saúde Pública. 2009; 33(4): 534-43.

26. 26 Pelicioni PHS, Pereira MP, Lahr J, Gobbi LTB. Análise cinética e cinemática do levantar e andar em jovens e idosos. Rev Bras Ciênc Esporte. 2015; 37(3):237-44.

27. Fonseca LCS, Scheicher ME. Relação entre projeção do centro de massa e equilíbrio em idosos. Terapia Manual. 2012;10(50):440-43. 
28. Cordeiro LL, Cordeiro LL, Paula e Oliveira MP, Martins WR. Caracterização das quedas em idosos e a relação mobilidade da coluna lombar e membros inferiores. Universitas: Ciências da Saúde. 2011;9(2):1-18.

29. Barry E, Galvin R, Keogh C, Horgan F,Fahey T.Is the Timed Up and Go test a useful predictor of risk of falls in community dwelling older adults: a systematic review and meta- analysis. BMC Geriatr.2014;14(14):1-14.

30. Greene B, Doheny EP, Walsh C, Cunningham C, Crosby L, Kenny RA. Evaluation of Falls Risk in Community Dwelling Older Adults Using Body-Worn Sensors. Gerontology. 2012;58(1):472-80.

Recebido em: 23/12/2018

Aceito em: 06/06/2019 